\title{
PRAXIS
}

\section{ESTUDIO DE CASO A UN USUARIO CON DIAGNÓSTICO: POST - OPERADO DE CRANEOTOMÍA DESCOMPRESIVA, PROBABLE EMPIEMA SUBDURAL}

Case study of a user with diagnosis: post-operated decompresive craniotomy, probable subdural empyema

SANDOVAL GUTIÉRREZ KARLA ABIGAIL*

ROJAS VEGA MARÍA EMMA**

\section{RESUMEN:}

ntroducción: el estudio de caso se centra en un usuario con diagnóstico médico post - operado de craneotomía descompresiva, probable empiema subdural, hospitalizado en el servicio de Medicina interna en una institución hospitalaria pública del estado de Sonora. Metodología: se le realizó una valoración focalizada de enfermería implementado la guía de valoración de enfermería según tipología de patrones funcionales de salud de Marjory Gordon. Se identificaron los diagnósticos de enfermería a partir de las respuestas únicas del usuario, empelando la taxonomía II de la NANDA Internacional y posteriormente se planearon las intervenciones, implementado planes de cuidado individualizados con las taxonomías NOC - NIC, enfocados a las necesidades del usuario. Resultados: se identificaron 8 diagnósticos de enfermería a partir de la valoración focalizada llevada a cabo, de los cuales cuatro son diagnósticos reales y cuatro son diagnósticos de riesgo. Conclusiones: la valoración de enfermería nos permite identificar las necesidades únicas de los usuarios y es responsabilidad del personal de enfermería tener los conocimientos necesario para poder brindar un cuidado humano con calidez y calidad a los usuarios con distintas patologías.

\section{ABSTRACT}

Introduction: The case study focuses on a user with post-operative medical diagnosis of decompressive craniotomy, probable subdural empyema, hospitalized in the Internal Medicine senvice in a public hospital institution in the state of Sonora. Methodology: A focused nursing assessment was carried out, implementing the nursing assessment guide according to the functional health pattern of Marjory Gordon. Nursing diagnoses were identified based on the unique responses of the user, using the NANDA - I taxonomy II; later the interventions were planned, implementing individualized care plans with the NOC - NIC taxonomies, focused on the person's needs. Results: Eight nursing diagnoses were identified from the focused assessment carried out, of which four are real diagnoses and four are risk diagnoses. Conclusions: the nursing assessment allows us to identify the unique users' needs and it is the nursing staff responsibility to have the necessary knowledge to be able to offer a warmth and quality human care to the users with different pathologies.

Key words: Nursing process, case studies, subdural hematoma, epilepsy, subdural empyema, craniotomy.

*Pasante de Servicio Social. Licenciatura en Enfermería. Departamento de Enfermería. Universidad de Sonora. Correo electrónico: sandovalabigail@gmail.com

**Pasante de Servicio Social. Licenciatura en Enfermería. Departamento de Enfermería Universidad de Sonora.
Palabras clave: Proceso de enfermería, estudio de caso, hematoma subdural, epilepsia, empiema subdural, craneotomía (DeCS, BIREME). 


\section{INTRODUCCIÓN}

El proceso de enfermería es un método sistemático, el cual nos permite administrar los cuidados de enfermería, a partir de las respuestas únicas de los usuarios, ante las alteraciones de salud reales y/o potenciales. Su objetivo es la conservación, recuperación o fomento de la salud de los usuarios, mediante la planeación de intervenciones enfocadas en las necesidades únicas de las personas. Es una herramienta cíclica, dinámica, racional y organizada, compuesta por 5 etapas: Valoración, diagnóstico, planeación, ejecución y evaluación (1).

Un estudio de caso es un fenómeno que se estudia en un contexto real, empelando diferentes fuentes de evidencia. Considerado como una valiosa herramienta de investigación, debido a que nos permite aprender la realidad de una situación (2). También es definido como una situación compleja y única que ocurre en la realidad. Así como una recolección y análisis formal de datos de un caso único ${ }^{(3)}$.

Ahora bien adentrándonos al estudio de caso expuesto; el heamtoma subdural es una acumulación de un volumen de sangre situado entre la duramadre y aracnoides (4). La craneotomía descompresiva es un procedimiento quirúrgico, en el cual se elimina un fragmento óseo del cráneo permitiendo la descompresión externa, favoreciendo el espacio de acomodación cerebral ${ }^{(5)}$.

El siguiente estudio de caso es aplicado a un usuario con Dx Hematoma subdural frontotempoparietal derecho + Hemorragia intraparenquimatosa frontal derecha. PO Craneotomía descompresiva, traqueostomía y gastrostomía / Epilepsia / Probable empiema subdural, hospitalizado en el servicio de Medicina interna en una institución hospitalaria pública del estado de Sonora. Con el propósito de llevar a cabo un análisis de dicho caso, que nos permita identificar los principales diagnósticos de enfermería, para implementar un plan de cuidado individualizado.

\section{METODOLOGÍA}

El presente caso fue elaborado en el servicio de Medicina interna el día 08 de septiembre de 2016 en una institución hospitalaria pública del estado de Sonora. Mediante la metodología del proceso de enfermería se realizó la valoración al usuario J.L.P.C., la recolección de información se llevó a cabo utilizando la guía de valoración de enfermería según tipología de patrones funcionales de salud de Marjory Gordon para persona adulta. Se implementó el interrogatorio indirecto a familiares del usuario debido a las condiciones de salud que presentaba, y se recopiló la información necesaria del expediente clínico.

Posterior al análisis - síntesis de los datos recolectados, identificamos los diagnósticos de enfermería presentes en el usuario a partir de las respuestas únicas de salud, utilizando la taxonomía II de la NANDA - I. Identificados los diagnósticos de enfermería, se realizó la planeación de las intervenciones de enfermería empleando las taxonomías $\mathrm{NOC}$ - NIC, que nos permitieron mantener o mejorar la salud y bienestar del usuario.

\section{PRESENTACIÓN DEL CASO}

\section{Datos sociales básicos del usuario}

J.L.P.C., hombre de 49 años, ingresa a una institución hospitalaria pública del estado de Sonora al servicio de Urgencias el día 21 de julio de 2016 con Dx Hematoma subdural frototempoparietal derecho + Hemorragia intraparenquimatosa frontal derecha. El usuario reside en $\mathrm{Mu}$ latos Sahuaripa, Sonora; familiares refieren que el motivo de su hospitalización fue por una cefalea intensa.

\section{Antecedentes personales}

Patológicos: Padece Epilepsia desde hace 18 años secundaria a cisticercosis con tratamiento médico de lamotrigina 1 tableta vía oral c/12 horas y valproato semisódico 500 $\mathrm{mg} \mathrm{c} / 12$ horas.

Heredo familiares: Padre finado por cáncer desde hace 30 años sin especificar, hermanos padecen hipertensión arterial.

\section{Resumen de inicio y evolución del padecimiento actual}

J.L.P.C., hombre de 49 años, el día 20 de julio del 2016, el usuario presenta cefalea de intensidad 10 - 10 acompañado de vómito y posteriormente crisis convulsivas tónico - clónico generalizada sin recuperación del estado de alerta, acude a valoración médica particular donde se inicia manejo de Midazolam $5 \mathrm{mg}$ y DFH $1 \mathrm{gr}$. Se refiere a otra unidad médica ingresando el día 21 de julio de 2016, nivel de escala de Glasgow \#5 con apertura ocular nula, verbal nulo, movimientos de decorticación, pupilas midriáticas sin respuesta a la luz, Dx Hematoma subdural frontotempoparietal derecho + Hemorragia intraparenquimatosa frontal derecha. Se realizó la valoración del estado de salud el día 08 de septiembre de 2016 con Dx Hematoma subdural frontotempoparietal derecho $+\mathrm{He}-$ morragia intraparenquimatosa frontal derecha. PO Craneotomía descompresiva, traqueostomía y gastrostomía / Epilepsia / Probable empiema subdural.

\section{Indicaciones médicas:}

-Dieta artesanal.

-Signos vitales por turno.

-Vigilancia neurológica.

-Cambios de posición.

-Fisioterapia. 


\begin{tabular}{|c|c|c|c|}
\hline Medicamento & Dosis & $\begin{array}{c}\text { Vía de } \\
\text { administración }\end{array}$ & Frecuencia \\
\hline Complejo B & 1 tableta & SGT & c/24 horas \\
\hline DFH & $100 \mathrm{mg}$ & SGT & c/8 horas \\
\hline Paracetamol & $1 \mathrm{~g}$ & IV & c/6 horas \\
\hline ASA & $100 \mathrm{mg}$ & SGT & c/24 horas \\
\hline Naproxeno & 2 tabletas & SGT & PRN \\
\hline Citicolina & $500 \mathrm{mg}$ & IV & Por las noches \\
\hline Lactulosa & $20 \mathrm{ml}$ & SGT & c/6 horas \\
\hline Nebulizaciones simples & $5 \mathrm{ml}$ & & $\mathrm{c} / 8$ horas \\
\hline Metronidazol & $500 \mathrm{mg}$ & $\mathrm{IV}$ & $\mathrm{c} / 12$ horas \\
\hline Vancomicina & $1 \mathrm{~g}$ vía & IV & $\mathrm{c} / 24$ horas \\
\hline Sirdalud (Tizandina) & $2 \mathrm{mg}$ & SGT & \\
\hline
\end{tabular}

Tabla 1. Resultados de bioquímica sanguínea del usuario en el Patrón Eliminación

\begin{tabular}{lcc}
\multicolumn{1}{c}{ Parámetros } & Resultados & Valor de referencia \\
\hline Creatinina & 0.68 & $0.7-1.2 \mathrm{mg} / \mathrm{dl}$ \\
Ácido úrico & 3.3 & $3.4-7 \mathrm{mg} / \mathrm{dl}$ \\
Sodio & 134 & $136-146 \mathrm{mEq} / \mathrm{ml}$ \\
Magnesio & 1.47 & $1.58-2.25 \mathrm{mg} / \mathrm{dl}$ \\
\hline
\end{tabular}

Fuente: Expediente Clínico

Tabla 3. Resultados de biometría hemática y tiempos de coagulación del usuario en el Patrón Actividad - Ejercicio

\begin{tabular}{lcc}
\multicolumn{1}{c}{ Parámetros } & Resultados & Valor de referencia \\
\hline Plaquetas & 575 & $139.0-45010 \mathrm{x}^{3} / \mathrm{ul}$ \\
Tiempo de protrombina & 16.0 & $11.5-15.2 \mathrm{seg}$ \\
Índice internacional normalizado (INR) & 1.23 & $0.0-1.0$ \\
\hline
\end{tabular}

Fuente: Expediente Clínico 


\section{Valoración focalizada por patrones funcionales de Marjory Gordon}

\section{Patrón Nutricional - Metabólico}

Usuario ingiere tres comidas diarias, su dieta presenta un alto contenido en carbohidratos, proteínas, cereales y legumbres, pobre en vitaminas y minerales, lo cual no guarda relación con su peso y talla al presentar un IMC de 21.95 $\mathrm{kg} / \mathrm{m} 2$, de acuerdo a la NOM-043-SSA2-2012 ${ }^{(6)}$ en un parámetro normal.

Durante su estancia hospitalaria tiene una dieta artesanal administrada por gastrostomía, ingresos totales de 746 $\mathrm{ml}$ (6 horas), hipertermia tratada por medios físicos. Edema de miembro superior izquierdo fóvea + y de miembros inferiores fóvea ++. Úlcera por presión estadio III en región de glúteo izquierdo, coloración rosada, diámetro de 3 $\mathrm{cm}$ y profundidad de $2 \mathrm{~cm}$, ausencia de exudado; en región plantar derecha presencia de úlcera por presión estadio II, coloración rosada, diámetro de $3 \mathrm{~cm}$ con exudado seroso moderado y en región plantar izquierda dos úlceras por presión estadio II de $3.5 \mathrm{~cm}$ y $2 \mathrm{~cm}$ de diámetro, coloración rosada y ausencia de exudado, tratadas mediante curación con jabón quirúrgico, agua inyectable y pasta Lassar; uñas de pies con onicomicosis tratadas con UNESIA.

Cabeza asimétrica en región tempoparietal derecha (abultamiento), parálisis de hemicara derecha mostrando alteración del par craneal VII nervio facial y edema palpebral en ojo derecho. Traqueostomía con piel circúndate en adecuadas condiciones; abdomen blando y depresible, presencia de gastrostomía permeable y funcional en epigastrio, piel circúndate a la sonda con adecuada coloración sin signos de infección.

De acuerdo a datos de laboratorio presenta leucocitosis (11600 uL) y neutrofilia (7400 uL) relacionado con proceso infeccioso en tratamiento con vancomicina y metronidazol (tabla 1). De acuerdo a escala de Norton obtiene una puntación de seis con riesgo alto de úlceras por presión, disminuyendo factores mediante uso de almohadillas y cambios de posición.

\section{Patrón Eliminación}

J.L.P.C. con evacuación intestinal en escala de Bristol \#4 con apoyo de Lactulosa. Evacuación urinaria de color amarillo claro mediante sonda vesical Foley Fr 18, diuresis horaria de $20.8 \mathrm{ml} /$ hora y diuresis kilogramo hora de $0.2 \mathrm{ml} / \mathrm{kg} /$ hora, tasa de filtrado glomerular de $130.1 \mathrm{ml} / \mathrm{min}$, con diminución de la producción de orina, obteniendo un balance + $376 \mathrm{ml}$ en 6 horas. De acuerdo a datos de laboratorio presente hiponatremia, hipomagnesemia, disminución de la creatinina y ácido úrico (tabla 2).

\section{Patrón Actividad - Ejercicio}

Usuario con polipnea e hipertensión arterial, y disminuyen posterior a normalización de temperatura, saturación de oxigeno de 99\%. Traqueostomía funcional en pieza en T a 5 L/min y FiO2 41\%; estertores en campos pulmonares, disminuyen posterior a aspiración de secreciones. Dependiente para realizar actividades de autocuidado; postura de decorticación con pie péndulo, espasmos musculares espontáneos con rigidez muscular de miembros superiores e inferiores en tratamiento con sirdalud y fisioterapia.

De acuerdo a datos de laboratorio presenta trombocitosis y alargamiento del tiempo de protrombina relacionado con tratamiento de anticoagulante plaquetario (tabla 3 y 4). De acuerdo a escala de Downton obtiene una puntuación siete con riesgo alto de caídas.

\section{Patrón Cognitivo - Perceptual}

Usuario con parpadeo espontáneo de ojo izquierdo sin alteración, ojo derecho con edema palpebral y sin posibilidad de parpadeo espontáneo. Pupilas midriáticas isocóricas, sin reacción a la luz y a la acomodación. Hipoacusia referida por familiar en oído izquierdo sin confirmar en examen físico. Incapacidad para hablar, reactivo a estímulos dolorosos, despierto pero indiferente al medio que lo rodea; alteración de par craneal III nervio oculomotor y par craneal VII nervio facial; presencia de signo meníngeo rigidez de nuca. Escala de Glasgow de ocho traumatismo craneoencefálico grave, escala de FOUR obtiene una puntuación de 11 y triada de Cushing negativa. 


\begin{tabular}{|c|c|}
\hline \multicolumn{2}{|c|}{$\begin{array}{c}\text { Diagnósticos de enfermería extraídos de NANDA - I (7), clasificados por patrones funcio- } \\
\text { nes de salud de M. Gordon en orden de prioridad }\end{array}$} \\
\hline Patrón funcional & Etiqueta diagnóstica \\
\hline Actividad - Ejercicio & $\begin{array}{l}\text { (00031) Limpieza ineficaz de las vías aéreas } \mathrm{r} / \mathrm{c} \text { vía aérea artifi- } \\
\text { cial (traqueostomía) y retención de secreciones } \mathrm{m} / \mathrm{p} \text { estertores } \\
\text { en campos pulmonares. }\end{array}$ \\
\hline Nutricional - Metabólico & $\begin{array}{l}\text { (00007) Hipertermia } r / c \text { enfermedad (probable empiema sub- } \\
\text { dural) } \mathrm{m} / \mathrm{p} \text { aumento de la temperatura corporal por encima del } \\
\text { límite normal }\left(38.1^{\circ} \mathrm{C} \text { ), taquicardia y calor al tacto. }\right.\end{array}$ \\
\hline Nutricional - Metabólico & $\begin{array}{l}\text { (00044) Deterioro de la integridad tisular r/c factores mecáni- } \\
\text { cos (presión) y deterioro de la movilidad física m/p destrucción } \\
\text { tisular (úlceras por presión estadio III en glúteo izquierdo). }\end{array}$ \\
\hline Nutricional - Metabólico & $\begin{array}{l}\text { (00046) Deterioro de la integridad cutánea } \mathrm{r} / \mathrm{c} \text { factores mecá- } \\
\text { nicos (presión) y prominencias óseas } \mathrm{m} / \mathrm{p} \text { destrucción de las } \\
\text { capas de la piel (úlceras por presión estadio II en región plantar } \\
\text { de miembro inferiores). }\end{array}$ \\
\hline Actividad - Ejercicio & $\begin{array}{l}\text { (00201) Riesgo de perfusión tisular cerebral ineficaz f/r trauma- } \\
\text { tismo cerebral. }\end{array}$ \\
\hline Actividad - Ejercicio & $\begin{array}{l}\text { (00040) Riesgo de síndrome de desuso f/r reducción de la mo- } \\
\text { vilidad. }\end{array}$ \\
\hline Cognitivo - Perceptual & $\begin{array}{l}\text { (00219) Riesgo de ojo seco f/r falta de parpadeo espontáneo } \\
\text { de ojo derecho. }\end{array}$ \\
\hline Actividad - Ejercicio & $\begin{array}{l}\text { (00155) Riesgo de caídas } \mathrm{f} / \mathrm{r} \text { deterioro de la movilidad física y } \\
\text { escala de Downton \#7 con riesgo alto de caídas. }\end{array}$ \\
\hline
\end{tabular}


Dominio: (11) Seguridad / Protección

Clase: (02) Lesión física

Diagnóstico enfermero (NANDA) ${ }^{(7)}$ : (00031) Limpieza ineficaz de las vías aéreas r/c vía aérea artificial (traqueostomía) y retención de secreciones $\mathrm{m} / \mathrm{p}$ estertores en campos pulmonares.

Objetivo: El usuario mantendrá permeabilidad de las vías aéreas, posterior a la aspiración de secreciones, durante el turno por la estudiante de enfermería.

Dominio: (II) Salud fisiológica Clase: (E) Cardiopulmonar

Resultado esperado (NOC) ${ }^{(8)}$ : (0410) Estado respiratorio: Permeabilidad de las vías respiratorias

Definición: Vías traqueobronquiales abiertas, despejadas y limpias para el intercambio de aire.

\begin{tabular}{|c|c|c|c|c|c|c|c|}
\hline \multirow[t]{2}{*}{ Indicadores } & \multicolumn{2}{|c|}{ Puntuación Diana } & \multicolumn{5}{|c|}{ Escala (s) de medición } \\
\hline & $\begin{array}{l}\text { Mante- } \\
\text { ner a: }\end{array}$ & $\begin{array}{l}\text { Aumen- } \\
\text { tar a: }\end{array}$ & 1 & 2 & 3 & 4 & 5 \\
\hline $\begin{array}{l}41004 \text { - Frecuencia } \\
\text { respiratoria }\end{array}$ & 4 & 5 & $\begin{array}{l}\text { Desvia- } \\
\text { ción grave }\end{array}$ & $\begin{array}{c}\text { Des- } \\
\text { viación }\end{array}$ & $\begin{array}{l}\text { Desviación } \\
\text { moderada }\end{array}$ & $\begin{array}{l}\text { Desvia- } \\
\text { ción leve }\end{array}$ & $\begin{array}{l}\text { Sin des- } \\
\text { viación }\end{array}$ \\
\hline $\begin{array}{l}41007-\text { Ruidos } \\
\text { respiratorios pato- } \\
\text { lógicos }\end{array}$ & 2 & 4 & $\begin{array}{c}\text { del rango } \\
\text { normal }\end{array}$ & $\begin{array}{l}\text { sustancial } \\
\text { del rango } \\
\text { normal }\end{array}$ & $\begin{array}{c}\text { del rango } \\
\text { normal }\end{array}$ & $\begin{array}{c}\text { del rango } \\
\text { normal }\end{array}$ & $\begin{array}{c}\text { del rango } \\
\text { normal }\end{array}$ \\
\hline $\begin{array}{l}41012 \text { - Capacidad } \\
\text { para eliminar se- } \\
\text { creciones }\end{array}$ & 2 & 3 & Grave & Sustancial & Moderado & Leve & Ninguno \\
\hline $\begin{array}{l}41020 \text { - Acumula- } \\
\text { ción de esputos }\end{array}$ & 2 & 4 & & & & & \\
\hline
\end{tabular}

\begin{tabular}{ll}
\hline \multicolumn{1}{c}{ Intervenciones (NIC) / Actividades ${ }^{(9)}$} & \multicolumn{1}{c}{ Fundamentación científica } \\
\hline $\mathbf{3 1 6 0}$ - Aspiración de vías aéreas: & $\begin{array}{l}\text { El aclaramiento fisiológico de la mucosidad de las } \\
\text { vías aéreas incluye el barrido ciliar y la tos. Su fra- } \\
\text { caso condiciona la acumulación de moco, la obs- } \\
\text {-Determinar la necesidad de la aspiración. }\end{array}$ \\
$\begin{array}{l}\text {-Auscultar ruidos respiratorios antes y después de la aspiración (esterto- } \\
\text { res con predominio en región apical y media). } \\
\text { aérea, el incremento del trabajo respiratorio, la ven- } \\
\text { tilación alveolar defectuosa, el desequilibrio venti- } \\
\text {-Realizar lavado de manos antes y después del procedimiento. }\end{array}$ & $\begin{array}{l}\text { lación perfusión y la mala oxigenación. El tiempo de } \\
\text { aspiraciones de } 10 \text { seg es el tiempo máximo para } \\
\text { evitar hipoxemia o atelectasia. Los movimientos } \\
\text {-Aspiración de secreciones con técnica abierta. }\end{array}$ \\
-Monitorizar estado de oxigenación del paciente (SaO2 99\%). & provocando un traumatismo el catéter tire de los tejidos
\end{tabular}
escasas y espesas).

\section{0 - Monitorización respiratoria:}

- Vigilar frecuencia, ritmo y profundidad de las respiraciones (FR de 22 por minuto, regulares y ligeramente profundas).

-Vigilancia de movimiento torácico (Expansión torácica simétrica).

-Aplicar sensores de oxigeno continuos no invasivos.

\section{Evaluación}

Posterior de la aspiración de secreciones la frecuencia respiratoria del usuario pasa de una puntuación diana de 4 a 5 (FR pasa de 22 a 20 por minuto). Los ruidos respiratorios y acumulación de esputo pasan de sustancial a leve, capacidad de eliminar se mantiene en sustancial. 
Dominio: (11) Seguridad / Protección

Clase: (06) Termorregulación

Diagnóstico enfermero (NANDA) ${ }^{(7)}$ : (00007) Hipertermia $\mathrm{r} / \mathrm{c}$ enfermedad (probable empiema subdural) $\mathrm{m} / \mathrm{p}$ aumento de la temperatura corporal por encima del límite normal $\left(38.1^{\circ} \mathrm{C}\right)$, taquicardia y calor al tacto.

Objetivo: El usuario disminuirá la hipertermia, posterior al uso de medios físicos y vigilancia de curva térmica, en un lapso de 2 horas.

Dominio: (II) Salud fisiológica Clase: (I) Regulación metabólica

Resultado esperado (NOC) ${ }^{(8)}$ : (0800) Termorregulación

Definición: Equilibrio entre la producción, la ganancia y la pérdida de calor.

\begin{tabular}{|c|c|c|c|c|c|c|c|}
\hline \multirow[t]{2}{*}{ Indicadores } & \multicolumn{2}{|c|}{$\begin{array}{l}\text { Puntuación } \\
\text { Diana }\end{array}$} & \multicolumn{5}{|c|}{ Escala (s) de medición } \\
\hline & $\begin{array}{c}\text { Mantener } \\
\text { a: }\end{array}$ & $\begin{array}{c}\text { Aumentar } \\
\text { a: }\end{array}$ & 1 & 2 & 3 & 4 & 5 \\
\hline $\begin{array}{l}80001 \text { - Temperatu- } \\
\text { ra cutánea aumen- } \\
\text { tada }\end{array}$ & 3 & 4 & & & & & \\
\hline $\begin{array}{l}80012 \text { - Frecuencia } \\
\text { del pulso radial }\end{array}$ & 2 & 4 & Grave & $\begin{array}{l}\text { Sustan- } \\
\text { cial }\end{array}$ & Moderado & Leve & Ninguno \\
\hline 80019 - Hipertermia & 3 & 4 & & & & & \\
\hline
\end{tabular}

\begin{tabular}{l} 
Intervenciones (NIC) / Actividades ${ }^{(9)}$ \\
\hline $\mathbf{3 9 0 0}$ - Regulación de la temperatura: \\
-Comprobar la temperatura al menos cada 2 horas. \\
-Observar el color y temperatura de la piel (calor al \\
tacto).
\end{tabular}

3740 - Tratamiento de la fiebre:

- Controlar la temperatura.

- Aplicar compresas húmedas tibias para disminuir la temperatura.

-Baño de esponja con agua tibita.

\section{0 - Monitorización de signos vitales:}

-Monitorizar pulso, temperatura y estado respiratorio. (Taquicardia FC 130 por minuto, temperatura de 38.1 ${ }^{\circ} \mathrm{C}$, hipertensión arterial 150/90 $\mathrm{mmHg}$ ).
Fundamentación científica

-La temperatura interna del cuerpo o central (boca, recto) es mayor que la de la superficie o periférica (axila o pliegue inguinal). La edad, medio ambiente, actividad muscular, metabolismo y condiciones patológicas son factores que influyen en las variaciones de la temperatura.

-La temperatura corporal en estado normal permanece constante por el equilibrio entre el calor producido (termogénesis) y el calor perdido (termólisis) ${ }^{(10)}$.

\begin{tabular}{l} 
Evaluación \\
\hline Usuario disminuye la temperatura $\left(37.8^{\circ} \mathrm{C}\right)$ pos- \\
terior a dos horas con el uso de compresas hú- \\
medas y posterior al baño disminuye dentro del \\
rango normal $\left(37.5^{\circ} \mathrm{C}\right)$. Temperatura cutánea e \\
hipertermia pasan de una puntuación diana de \\
3 a 4 y la frecuencia del pulso pasa de una pun- \\
tuación diana de 2 a 4 . (Días posteriores de la \\
valoración usuario se encuentra normotérmico).
\end{tabular}


Dominio: (04) Actividad / Reposo

Clase: (04) Respuestas cardiovasculares / pulmonares

Diagnóstico enfermero (NANDA) ${ }^{(7)}$ : (00201) Riesgo de perfusión tisular cerebral ineficaz f/r traumatismo cerebral.

Objetivo: El usuario disminuirá el riesgo de perfusión tisular cerebral, posterior a la monitorización neurológica, durante su estancia hospitalaria.

Dominio: (II) Salud fisiológica

Clase: E-Cardiopulmonar

Resultado esperado (NOC) ${ }^{(8)}$ : (0406) Perfusión tisular: Cerebral.

Definición: Adecuación del flujo sanguíneo a través de los vasos cerebrales para mantener la función cerebral.

\begin{tabular}{|c|c|c|c|c|c|c|c|}
\hline \multirow[t]{2}{*}{ Indicadores } & \multicolumn{2}{|c|}{$\begin{array}{l}\text { Puntuación } \\
\text { Diana }\end{array}$} & \multicolumn{5}{|c|}{ Escala (s) de medición } \\
\hline & $\begin{array}{c}\text { Mantener } \\
\text { a: }\end{array}$ & $\begin{array}{c}\text { Aumentar } \\
\text { a: }\end{array}$ & 1 & 2 & 3 & 4 & 5 \\
\hline $\begin{array}{l}40618 \text { - Deterioro } \\
\text { cognitivo }\end{array}$ & 2 & 3 & & & & & \\
\hline $\begin{array}{l}40619 \text { - Nivel de } \\
\text { conciencia dismi- } \\
\text { nuido }\end{array}$ & 2 & 3 & Grave & $\begin{array}{l}\text { Sustan- } \\
\text { cial }\end{array}$ & Moderado & Leve & Ninguno \\
\hline $\begin{array}{l}40620-\text { Reflejos } \\
\text { neurológicos alte- } \\
\text { rados }\end{array}$ & 1 & 2 & & & & & \\
\hline
\end{tabular}

Intervenciones (NIC) / Actividades (9) 2620 - Monitorización neurológica:

- Comprobar tamaño, forma, simetría y capacidad de reacción de las pupilas (midriáticas $6 \mathrm{~mm}$, isocóricas, redondas, sin reaccionan a la luz).

-Comprobar nivel de orientación (Despierto pero indiferente al medio que lo rodea).

- Vigilar Escala de Glasgow (\#8 traumatismo craneoencefálico grave).

-Monitorizar simetría facial (Asimetría facial).

-Comprobar respuesta a estímulos (Responde a estímulos dolorosos).

-Monitorización de signos vitales.

- Vigilar características del habla (Ausencia del habla).

\section{Fundamentación científica}

El control neurológico se define como el procedimiento exploratorio que nos da a conocer el estado neurológico de un paciente y su evolución ${ }^{(11) .}$

\section{Evaluación}

Los indicadores de deterioro cognitivo y nivel de conciencia disminuido, permanecen en una puntuación diana de 2 , y reflejos neurológicos alterados permanece en una puntuación diana de 1 . 


\section{CONCLUSIONES}

La valoración de enfermería nos permite identificar las necesidades únicas de los usuarios y es responsabilidad del personal de enfermería tener los conocimientos necesario para poder brindar un cuidado humano con calidez y calidad a los usuarios con distintas patologías. En el estudio de caso expuesto, era de suma importante hacer una valoración minuciosa de las respuestas únicas del usuario, ya que nos permitió priorizar el cuidado que debemos brindarle, implementando planes de cuidado individualizados, favoreciendo al mantenimiento y recuperación del estado de salud. Podemos apreciar, como un evento que perturbó la salud del usuario, desencadenó otras alteraciones, que cambiaron su funcionalidad y prolongaron su estancia hospitalaria. La relevancia de este estudio para la profesión de enfermería, recae en ser un estudio de caso único, ya que en cada una de las patologías mencionadas se conoce la sintomatología general, pero los usuarios manifiestan respuestas únicas ante la enfermedad, por lo tanto no todos tendrán la misma manifestación. Por lo tanto, se apreció la transición del estado de salud - enfermedad del usuario, que nos servirá como pauta para futuros casos que puedan presenciar los profesionales de la salud.

\section{REFERENCIAS BIBLIOGRÁFICAS}

1. Kozier B, Berman A, Snyder S, Erb G. Fundamentos de enfermería: conceptos, proceso y práctica. Vol. 2. 8a ed. Madrid, España: PEARSON EDUCACIÓN, S.A.; 2008.

2. Jiménez VE. El estudio de caso y su implementación en la investigación. Rev. Int. Investig. Cienc. Soc. 2012; 8(1):141-50.

3. Monroy SH. El Estudio De Caso: ¿Método o Técnica de Investigación? Revista de la Asociación Mexicana de Metodología de la Ciencia y de la Investigación. 2009; 1(1):38-64.

4. Mosquera BG, Varela HA. Traumatismo craneoencefálico en el adulto. Temas para la Atención Primaria de Salud. La Habana: Ciencias Médicas; 2011.

5. Lacerda AJ. Craniectomía descompresiva en el tratamiento del traumatismo craneoencefálico grave. Rev. Cubana Neurol. Neurocir. 2013; 3(1):93-100.

6. Norma Oficial Mexicana NOM-043-SSA2-2012, Servicios básicos de salud. Promoción y educación para la salud en materia alimentaria. Criterios para brindar orientación. Diario Oficial de la Federación, (22-01-2013). 7. Herdman TH. NANDA Internacional. Diagnósticos Enfermeros: definición y clasificación. 2015-2017. Barcelona: Elsevier; 2015.

8. Moorhead S, Johnson M, Maas M, Swanson E. Clasificación de resultados de enfermería (NOC). 5 a ed. España: Elsevier; 2014.
9. Bulechek G, Bulecher H, Dochterman J, Wagner C. Clasificación de intervenciones de enfermería (NIC). $6 a$ ed. España: Elsevier; 2013.

10. Reyes E. Fundamentos de enfermería: ciencia, metodología y tecnología. 1a ed. México: El Manual Moderno; 2009.

11. Carmona JV, Martínez ME, Gallego JM, Castaño M. El control neurológico en el paciente crítico: Valoración de enfermería. Revista de Enfermería Integral. 2000. 